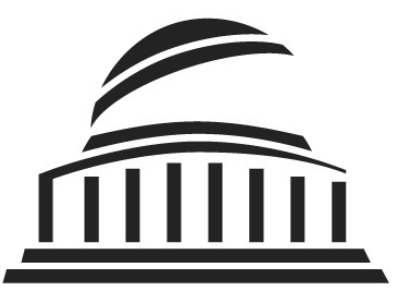

MIT
Open Access Articles

\title{
Molecular Dynamics-Based Simulation on Chemical Flooding Produced Emulsion Formation and Stabilization: A Critical Review
}

The MIT Faculty has made this article openly available. Please share how this access benefits you. Your story matters.

\begin{tabular}{|l|l|}
\hline As Published & https://doi.org/10.1007/s13369-020-04840-9 \\
\hline Publisher & Springer Berlin Heidelberg \\
\hline \hline Version & Author's final manuscript \\
\hline Citable link & https://hdl.handle.net/1721.1/131563 \\
\hline Terms of Use & $\begin{array}{l}\text { Article is made available in accordance with the publisher's } \\
\text { policy and may be subject to US copyright law. Please refer to the } \\
\text { publisher's site for terms of use. }\end{array}$ \\
\hline
\end{tabular}




\section{Molecular Dynamics-Based Simulation on Chemical Flooding Produced Emulsion Formation and Stabilization: A Critical Review}

Cite this article as: Zhihua Wang, Yunfei Xu, Yang Liu, Xiaoyu Liu and Zhenhua Rui, Molecular Dynamics-Based Simulation on Chemical Flooding Produced Emulsion Formation and Stabilization: A Critical Review, Arabian Journal for Science and Engineering https://doi.org/10.1007/s13369-020-04840-9

This Author Accepted Manuscript is a PDF file of an unedited peer-reviewed manuscript that has been accepted for publication but has not been copyedited or corrected. The official version of record that is published in the journal is kept up to date and so may therefore differ from this version.

Terms of use and reuse: academic research for non-commercial purposes, see here for full terms. https://www.springer.com/aam-terms-v1 


\title{
Molecular Dynamics-Based Simulation on Chemical Flooding Produced
}

\section{Emulsion Formation and Stabilization: A Critical Review}

\author{
Zhihua Wang $^{1} \cdot$ Yunfei Xu $^{1} \cdot$ Yang Liu $^{1} \cdot$ Xiaoyu Liu ${ }^{1} \cdot$ Zhenhua Rui $^{2, *}$ \\ ${ }^{1}$ Key Laboratory for Enhanced Oil \& Gas Recovery of the Ministry of Education, Northeast Petroleum \\ University, Daqing, Heilongjiang, 163318, PR China; zhihua_wang@126.com (Zhihua Wang); \\ xuyunfei103@163.com (Yunfei Xu); lynepu@163.com (Yang Liu); lxyljy160930@163.com (Xiaoyu Liu) \\ ${ }^{2}$ Massachusetts Institute of Technology, Cambridge, MA 02139, USA; zhenhuarui@gmail.com (Zhenhua \\ Rui) \\ "Corresponding author: zhenhuarui@gmail.com
}

\begin{abstract}
Oil-water emulsions are commonly encountered at various stages of petroleum production. For example, the alkaline-surfactant-polymer (ASP) flooding is extensively used to promote emulsion formation, and thus, enhance oil recovery (EOR). However, the chemicals (e.g. polymers and surfactants) involved in this process can also stabilize the produced emulsions to adversely affect the subsequent processes of oil field surface systems. Therefore, a deep understanding of oil-water emulsions formation and stabilization is required to guarantee and promote oil field production. This work summarizes the current knowledge on (i) the formation of oil-water emulsions, (ii) the influence of crude oil components (e.g. asphaltenes and resins), and (iii) the above-mentioned water phase components on emulsions stability on a macroscopic scale. Moreover, considering the importance of molecular dynamics (MD) simulation for revealing interphase interactions and its advantages of microstructure characterization, we also probe the mechanism of such simulations, discuss the obtained results, and reveal progress in the elucidation of the mechanism of oil-water interface stabilization. MD simulation is shown to shed light on oil-water emulsification and demulsification processes and is concluded to be well suited for exploring molecular adsorption, droplet coalescence, and droplet separation on a micro scale. However, future researchers should aim to circumvent the limitations of model simplification and single-factor simulation, integrate the characteristics of internal and external phase components, and consider external factors like temperature and pressure to comprehensively analyze crude oil emulsification and demulsification behavior. Furthermore, the potential role of bubbles on produced emulsion structure should be considered in future simulations.
\end{abstract}

Keywords: interface formation energy; radial distribution function; diffusion coefficient; produced emulsion; ASP flooding; molecular dynamics

\section{Introduction}

Although the adjustment and optimization of the global energy structure have decreased the rate of oil demand growth, the oil demand of major developing countries continues to increase, which makes oil recovery enhancement an important direction of oilfield development and construction [1,2]. In crude oil production and processing, oil-water separation, as the core of crude oil field processing, plays an important role in supporting projects aimed at enhanced 
crude oil recovery. However, oil-water emulsions have complex and changeable properties influenced by crude oil/water phase composition and the external environment, which shows that an understanding of factors affecting oil-water emulsion stability is important to ensure and promote the efficient operation of crude oil dehydration and sewage treatment technology in system of oil field surface production [3].

The popularization and implementation of the tertiary chemical flooding technology, based on the application of polymers, surfactants, and alkali as oil displacement agents, have significantly contributed to oil recovery enhancement $[4,5]$. At the same time, the above substances are recovery with the produced liquid and act on the oil-water interfacial film to affect the stability of oil-water emulsions [6,7]. Anionic surfactants commonly used in the alkaline-surfactant-polymer (ASP) flooding technology, e.g., petroleum sulfonates and heavy alkyl benzene sulfonates, feature a hydrophilic group at one end of the molecule connected to a hydrophobic tail, thus exhibiting amphiphilicity [8], which determines both aggregation behavior and directional adsorption arrangement at the interface. In addition, the dissociation of anionic surfactants in aqueous media affords negatively charged molecules and thus changes, the electrical properties of the oil-water interfacial film and hinders the coalescence of (similarly) charged water droplets to enhance emulsion stability [9,10]. Thus, the development of highly efficient demulsifiers and demulsification technologies as well as the realization of efficient oil-water separation are significant for the elucidation and multiple-aspect characterization of the origin of crude oil emulsion stability.

This review summarizes the current knowledge on the formation of oil-water emulsions and generalizes our understanding of the factors influencing the stability of oil-water emulsions on a macroscopic scale. The applicability of molecular dynamics (MD) simulation to microstructural characterization makes such simulations well suited for revealing interactions between phases. The mechanism of MD simulation, the obtained results, and the progress in the study of the stabilization mechanism of the oil-water interface are discussed to deepen our microscopic-scale understanding of phenomena determining the thickness of the oil-water interfacial film, the arrangement and aggregation behavior of molecules at the oil-water interface, and the stability of this interface. Research applications and future development directions of MD simulation methods in the field of oil-water emulsification and demulsification are put forward to provide a reference for enriching the theory of oil-water emulsification and demulsification and promoting the development and application of complex emulsion separation technologies.

\section{Formation and Stabilization of emulsions}

\subsection{Formation of crude oil emulsions}

All crude oils, regardless of their source, can form emulsions [11], which are defined as mixtures of two immiscible liquids in which the droplets of one liquid are stably suspended in the other liquid [12]. However, the size of emulsion increases with time to result in gravity-driven separation, i.e., emulsions are thermodynamically unstable. Therefore, to realize stable emulsions, one should slow down or fully hinder droplet coalescence through the addition of emulsifiers, that is, increase the kinetic stability of emulsions [13]. Emulsions are ubiquitous in crude oil production and can be broadly classified into water-in-oil (W/O), oil-in-water (O/W), and multilevel or complex emulsions [14].

\subsection{Stabilization of crude oil emulsions}

Crude oil emulsions are complex dispersion systems, the stability of which is determined by factors such as aging and the properties of oil (asphaltene content, resin content, solid particle content, density, viscosity, etc., and the properties of water phase, including water content, water droplet diameter, water droplet electrification, salinity, $\mathrm{pH}$, etc.) [15-17]. Facing the problem of the factors which the polar component of crude oil phase and chemistry component in water phase how to affect the emulsions stability, the interaction mechanism of the oil-water interface has mainly probed by macroscopic experiments for a long time, and some common understandings have been formed to reveal the behavior of oil-water interface. 


\subsubsection{Components of crude oil phase}

Asphaltenes. Asphaltenes, defined as the crude oil fraction insoluble in pentane, hexane, or heptane but soluble in toluene or benzene, are the most polar and largest-molecular-weight crude oil components, comprising several polynuclear aromatic sheets surrounded by hydrocarbon tails, forming particles with molar masses of 500-20,000 $g$ [18], and featuring strong lipophilicity and excellent emulsifying capacity. Therefore, these compounds easily adsorb at the oil-water interface to increase the strength of the oil-water interfacial film and, thus, emulsion stability. To study the effects of asphaltenes on emulsion stability, Dicharry [18] carried out a series of experiments with asphaltene-free crude oil, revealing that at asphaltene concentration levels of $<4 \%$, the produced films were mobile and weak, while much more robust films were produced at higher contents. Fingas [19] pointed out that stable emulsions can be formed at asphaltene contents above $7 \mathrm{wt} \%$, which, to some extent, agreed with the conclusion of Dicharry. Chen et al. [20] studied the interaction between emulsion droplets and interface-adsorbed asphaltenes by droplet-probe atomic force microscopy, showing that the stability of crude oil emulsions is related to asphaltene concentration, with interface-adsorbed asphaltenes not only hindering the coalescence of unbound water droplets in the crude oil phase, but also inducing interfacial adhesion during droplet separation. At low concentrations of $<100 \mathrm{mg} / \mathrm{L}$, adhesion increased with increasing asphaltene concentration, further decreasing at higher concentrations.

Resins. Resins, defined as the fraction soluble in light alkanes (pentane, hexane, or heptane) but insoluble in liquid propane, mainly comprise naphthenic aromatic hydrocarbons (generally aromatic ring systems with alicyclic chains) and are effective dispersants of asphaltenes in crude oil [18]. Resins feature lower molecular weight and polarity than asphaltenes and thus form less rigid films upon adsorption onto droplets [20]. Unlike in the case of asphaltenes, the influence of resins alone on emulsion stability has not been well studied, as scholars usually research the cumulative contribution of resin and asphaltene to the stability of emulsions at the same time. Andersen and Birdi [21] used thermal titration to determine the change of asphaltene critical micelle concentration due to resin addition to asphaltene-containing emulsions, revealing that resins can adsorb on asphaltenes to change the micelle shape of the latter and afford new micelles with decreased volume. The synergistic effect of resins and asphaltenes can increase the solubility of the latter species, prevent their accumulation and precipitation, facilitate the adsorption of asphaltene micelles on the oil-water interface, and enhance the stability of crude oil emulsions.

\subsubsection{Components of water phase}

Surfactants. Surfactants, i.e., substances with hydrophilic polar groups and lipophilic non-polar groups, do not satisfy the dual affinity criterion unless located at the water-oil phase interface, with the polar part immersed into water and the non-polar part immersed into oil [22]. Therefore, in oil-water systems, surfactants are generally adsorbed at the interface to reduce system free energy and form an interfacial film. The interfacial tension (IFT) is the change in free energy with an infinitesimal increase of the contact area between the two phases of interest. When surfactant is present, the interfacial tension could be reduced several orders of magnitude [23]. With increasing surfactant content, the tightness of the interfacial arrangement and the strength of the interfacial film increase. However, when the surfactant concentration reaches the critical micelle concentration, surface tension no longer decreases with increasing surfactant content because of micelle formation. In a study conducted by Hakiki et al [24], in order to build an ultra-low interfacial tension experimental environment, a mixture of anionic ethoxy carboxylate and non-ionic ethoxylate was selected after rigorous screening of dozens of surfactants, which hit a low IFT point at $0.0004119 \mathrm{mN} / \mathrm{m}$. It can also be considered a very low IFT value reported due to anionic and non-ionic surfactant addition. Meanwhile, numerous studies have proven that surfactants affect emulsion stability through electrostatic repulsion. Anionic surfactants can adsorb at the oil-water interface to form a double electric layer inside droplets and thus make them electrostatically repel each other. Studies of the effects of sodium dodecyl sulfate (SDS, an anionic surfactant) and cetyl trimethyl ammonium bromide (CTAB, a cationic surfactant) on the properties of nanoemulsions showed that emulsion stability increases upon the 
addition of the former and decreases in the presence of the latter, which confirms the large role of electrostatic repulsion in determining emulsion stability [25]. Moreover, a cationic surfactant was also introduced by Azadeh as a demulsifier [26]. In addition, due to the foaming characteristics of surfactants, the bubbles with different size could be generated in emulsions, and these bubbles would affect the emulsification behavior [6]. However, the definitive relationship between bubbles and emulsification stability has not been clarified.

Polymers. Li et al. [27] measured the interfacial shear viscosity, interfacial tension, zeta potential, and emulsion stability of crude oil, and studied the effect of hydrolyzed polyacrylamide (HPAM) on emulsion interface properties and stability, showing that HPAM can be adsorbed at the oil-water interface but has no effect on interfacial tension. With increasing HPAM concentration, the interfacial shear viscosity, zeta potential, and stability of oil-water emulsions increased. The phase transition combination method was used to compare the effects of HPAM and hydrophobically modified polyacrylamide (HMPAM) with different structures on the stability of water-oil emulsions [28], and the results revealed that both HPAM and HMPAM can stabilize emulsions. The amphiphilic HMPAM was found to form an intramolecular salt and be active at the interface, thus being more effective than HPAM. Moreover, because of being involved in a combined thickening mechanism, HMPAM can also increase emulsion viscosity, adsorb at the oil-water interface, and interact with surfactants to form a mixed solid adsorption layer and thus increase interfacial film strength. Meller and Stavans [29] studied the effect of hydrophilic polymers on the stability of oil-in-water emulsions, revealing that these compounds could increase the interfacial film strength of crude oil emulsions and hinder the coalescence of oil droplets. Unlike in the case of surfactants, studies probing the effects of polymers on the stability of crude oil emulsions are few, but their general conclusion is almost the same, i.e., polymer adsorption at the oil-water interface increases interfacial film strength and thickness to hinder droplet coalescence and enhance the stability of crude oil emulsions [30,31].

\section{Molecular dynamics simulation}

Although many studies have described the interaction between phases on a macroscopic scale and developed the theory of oil-water emulsification and stability, the behavior and influence of the oil-water interface still needs to be explored on a microscopic scale to enrich the theory of oil-water emulsification/demulsification, promote the development of high-efficiency demulsifiers and demulsification technologies, and facilitate the realization of highly efficient oil-water separation. The concept of MD simulation, first proposed by Alder and Wainwright in 1957 and used to study the interaction between hard spheres [32], has been widely used in the fields of physics, chemistry, biology, materials science, energy, etc [33-35]. MD simulations combine classical mechanics with quantum and statistical mechanics to describe the physical changes of various complex structures under different conditions. At present, this technique is widely used to model complex systems, as it provides a highly accurate description of particle motion according to Newton's laws of dynamics and allows the phase trajectories of a given molecular system to be obtained by numerical simulation, thus enabling the acquisition of thermodynamic data and system structural characteristics [36]. To understand the micromechanism and thermodynamics of phase transformations and the precipitation of waxy crystals in crude oil mixtures, we probed the phase transformation and gelling behavior of waxy molecules in the oil-asphaltene-water multiphase system by MD simulation [37], with the employed system model and related details shown in Fig. 1. The results shed light on the dissolution, diffusion, and aggregation of waxy crystals in crude oil mixtures and provided theoretical support for wax removal in waxy crude oil production systems. 

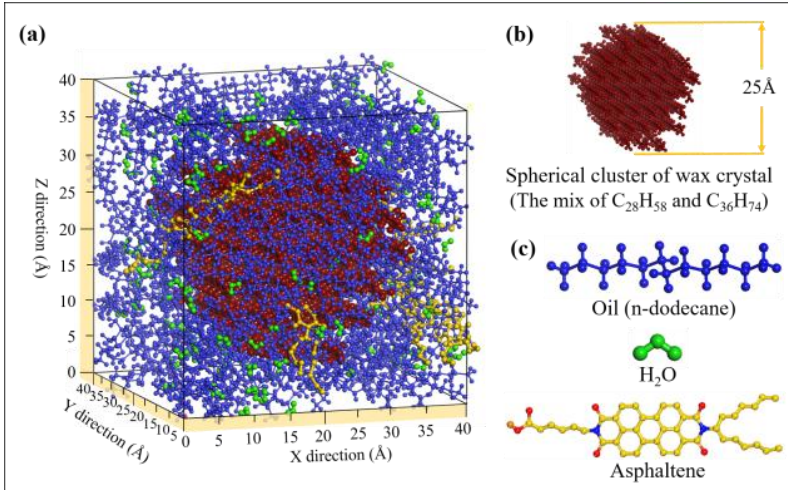

Fig.1 Simulated model of waxy crystals molecules in the crude oil mixture system [37]

\subsection{Mechanism of molecular dynamics simulation}

MD simulations employ the finite difference method, most commonly using Verlet, leap-frog, Beeman, and correction prediction algorithms [38].

Verlet algorithm. As the most simple and widely used MD algorithm, the Verlet algorithm uses the position $r(t)$ of a particle at time $t$, acceleration $a(t)$, and position at time $t-\delta t$ to calculate the position at time $t+\delta t$. Expansion of the particle position according to the Taylor formula affords

$$
r(t+\delta t)=r(t)+\frac{\mathrm{d}}{\mathrm{d} t} r(t) \delta t+\frac{1}{2 !} \frac{\mathrm{d}^{2}}{\mathrm{~d} t^{2}} r(t)(\delta t)^{2}+\cdots
$$

Substitution of $\delta t$ in the above equation with $-\delta t$ gives

$$
r(t-\delta t)=r(t)-\frac{\mathrm{d}}{\mathrm{d} t} r(t) \delta t+\frac{1}{2 !} \frac{\mathrm{d}^{2}}{\mathrm{~d} t^{2}} r(t)(\delta t)^{2}+\cdots
$$

Equation (1) subtracted Equation (2) to get the velocity formula

$$
v(t)=\frac{\mathrm{d} r}{\mathrm{~d} t}=\frac{1}{2 \delta t}[r(t+\delta t)-r(t-\delta t)]
$$

while the addition of the two equations gives

$$
r(t+\delta t)=-r(t-\delta t)+2 r(t)+\frac{\mathrm{d}^{2}}{\mathrm{~d} t^{2}} r(t)(\delta t)^{2}
$$

As $\frac{\mathrm{d}^{2}}{\mathrm{~d} t^{2}} r(t)=a(t)$, the position at $t+\delta t$ can be predicted from positions at $t$ and $t-\delta t$ by the above equation.

The Verlet algorithm features the advantages of execution simplicity and small memory demand but suffers from poor accuracy. In addition, it is not a self-starting algorithm, i.e., the new particle position must be obtained from positions at $t$ and $t-\delta t$. However, at $t=0$, there is only one group of positions, and the position at $t-\delta t$ has to be determined by other methods, which select an approximate value.

Leap-frog algorithm. In view of the shortcomings of the Verlet algorithm, Hockney proposed the related leap-frog algorithm, which determines position and speed as

$$
\left\{\begin{array}{l}
\vec{v}_{i}\left(t+\frac{1}{2} \delta t\right)=\vec{v}_{i}\left(t-\frac{1}{2} \delta t\right)+\vec{a}_{i}(t) \delta t \\
\vec{r}_{i}(t+\delta t)=\vec{r}_{i}(t)+\vec{v}_{i}\left(t+\frac{1}{2} \delta t\right)
\end{array}\right.
$$


This algorithm assumes that $\vec{v}_{i}\left(t+\frac{1}{2} \delta t\right)$ and $\vec{r}_{i}(t)$ are known, calculating the force acting on a particle and its acceleration $\vec{a}_{i}(t)$ from position $\vec{r}_{i}(t)$ at time $t$. Then velocity $\vec{v}_{i}\left(t+\frac{1}{2} \delta t\right)$ at time $t+\frac{1}{2} \delta t$ is predicted, and so on.

According to $\vec{v}_{i}\left(t+\frac{1}{2} \delta t\right)$ and $\vec{v}_{i}\left(t-\frac{1}{2} \delta t\right)$, speed at time $t$ can be obtained as

$$
\vec{v}_{i}(t)=\frac{1}{2}\left[\vec{v}_{i}\left(t+\frac{1}{2} \delta t\right)+\vec{v}_{i}\left(t-\frac{1}{2} \delta t\right)\right]
$$

Only two pieces of information, namely $\vec{v}_{i}\left(t-\frac{1}{2} \delta t\right)$ and $\vec{r}_{i}(t)$, need to be stored in the case of the leap-frog algorithm, which not only saves memory space, but also results in high accuracy and stability. The leap-frog algorithm does not need to calculate the next position to obtain velocity, but it should be noted that velocity is not defined at the same time as position, i.e., kinetic energy and potential energy are not defined at the same time, which precludes the direct calculation of total energy.

Beeman algorithm. The Beeman algorithm can be described as

$$
\left\{\begin{array}{l}
\vec{r}_{i}(t+\delta t)=\vec{r}_{i}(t)+\vec{v}_{i}(t) \delta t+\frac{1}{6}\left[4 \vec{a}_{i}(t)-\vec{a}_{i}(t-\delta t)\right] \delta t^{2} \\
\vec{v}_{i}(t+\delta t)=\vec{v}_{i}(t)+\frac{1}{6}\left[2 \vec{a}_{i}(t+\delta t)-5 \vec{a}_{i}(t)-\vec{a}_{i}(t-\delta t)\right] \delta t
\end{array}\right.
$$

This algorithm needs to store $\vec{r}_{i}(t), \vec{v}_{i}(t)$, and $\vec{a}_{i}(t-\delta t)$, thus featuring a larger storage capacity than the Verlet algorithm and offering the advantage of quoting a long integration interval. Specifically, the integration step $\delta t$ quoted by the Beeman algorithm can be 3-4 times that of the Verlet algorithm at the same level of accuracy.

Predictor-corrector algorithm. The continuity of a given particle's trajectory allows the position and velocity at time $t+\delta t$ to be predicted by Taylor expansion of time $t$ as

$$
\left\{\begin{array}{l}
\vec{r}^{p}(t+\delta t)=\vec{r}(t)+\vec{v}(t) \delta t+\frac{1}{2} \vec{a}(t) \delta t^{2}+\frac{1}{6} \vec{b}(t) \delta t^{3}+\cdots \\
\vec{v}^{p}(t+\delta t)=\vec{v}_{i}(t)+\vec{a}(t) \delta t+\frac{1}{2} \vec{b}(t) \delta t^{2}+\cdots \\
\vec{a}^{p}(t+\delta t)=\vec{a}(t)+\vec{b}(t) \delta t+\cdots \\
\vec{b}^{p}(t+\delta t)=\vec{b}(t)+\cdots
\end{array}\right.
$$

As these physical quantities originate from the Taylor expansion and not from the Newton equation of motion, the resulting velocity, acceleration, etc. are not completely correct. To solve this problem, one can use the predicted position $\vec{r}^{p}(t+\delta t)$ to calculate the force and the correct acceleration $\vec{a}^{p}(t+\delta t)$. Supposing that the difference between the correct acceleration and the predicted acceleration is given by

$$
\Delta \vec{a}(t+\delta t)=\vec{c}^{c}(t+\delta t)-\vec{a}^{p}(t+\delta t)
$$

the correction equation of each physical quantity can be obtained as 


$$
\left\{\begin{array}{l}
\vec{r}^{c}(t+\delta t)=\vec{r}^{p}(t+\delta t)+\mathrm{c}_{0} \Delta \vec{a}(t+\delta t) \\
\vec{v}^{c}(t+\delta t)=\vec{v}^{p}(t+\delta t)+\mathrm{c}_{1} \Delta \vec{a}(t+\delta t) \\
\vec{a}^{c}(t+\delta t)=\vec{a}^{p}(t+\delta t)+\mathrm{c}_{2} \Delta \vec{a}(t+\delta t) \\
\vec{b}^{c}(t+\delta t)=\vec{b}^{p}(t+\delta t)+\mathrm{c}_{3} \Delta \vec{a}(t+\delta t)
\end{array}\right.
$$

where $\mathrm{c}_{0}, \mathrm{c}_{1}, \mathrm{c}_{2}$, and $\mathrm{c}_{3}$ are constants.

This first-order predictor-corrector algorithm can be extended to a higher-order one. Generally, prediction accuracy increases with increasing order of Taylor expansion. However, this increase comes at the expense of substantially increased memory demand, and the above algorithm is therefore not as convenient as the Verlet algorithm for the simulation of a large number of molecular systems.

\subsection{Processes of molecular dynamics simulation}

\subsubsection{System establishment}

The first step of MD simulations is the construction of a suitable simulation system, with build quality directly affecting the accuracy of the obtained results [36]. In the case of an irrational build, it is difficult for the system to reach a stable equilibrium state, and prediction accuracy cannot be guaranteed. To ensure simulation stability, high-energy conformations should be avoided as much as possible. At present, many simulation programs such as Materials Studio [39] and GROMACS [40] can be used to build the initial model. In addition, appropriate force field parameters and ensemble/boundary conditions should be selected for initial model construction. Different force field parameters should be considered for different simulation systems, and force field limitations often directly affect prediction accuracy. As the accumulation of molecules may cause overlap and cross problems during modeling, the energy minimization method should be used for conformation optimization before calculations on the constructed initial system to reduce the generation of unreasonable conformations [40].

\subsubsection{Simulation calculation}

During simulation calculations, the initial state with a certain condition, namely the initial position, initial velocity, and force of all particles, is set first. Then, the spatial position, velocity, and acceleration of particles at the next moment are obtained by solving the motion equation, and the motion trajectories of all particles in the whole simulation system are counted until the system properties become stable [41]. Analysis of system structure and thermodynamic parameters allows one to determine whether the simulation has reached the equilibrium state. It is generally believed that this state can be reached when the structure, energy, temperature, pressure, and other thermodynamic parameters of the whole system no longer change with time, or change within 5\% [36].

\subsubsection{Data analysis}

The last step of MD simulation is the analysis and discussion of data obtained after equilibrium establishment. Notably, MD simulation allows one to obtain the positions and velocities of all particles in the system at each time point, i.e., the motion trajectory. A large amount of thermodynamic and statistical information can be extracted from the trajectory file, and the information related to the research object can be collected for processing, discussion, and analysis [41].

\subsection{Characterization of molecular dynamics simulation results}

\subsubsection{Density distribution}

The diagram of density distribution in each direction can be obtained by calculating the density distribution 
of the system in $\mathrm{X}, \mathrm{Y}$, and $\mathrm{Z}$ directions. Taking oil-water emulsions as an example, the distribution, equilibrium structure, and the thickness of interfacial films formed between different phases can be clearly identified from the diagram because of phase density differences. MD simulation has been used to study the microscale behaviors of four surfactants at the oil-water interface [42]. Specifically, density distribution diagrams were used to determine interface thickness and calculate the oil-water interface thicknesses of different surfactant systems (anion, non-ion, zwitterion, and gemini) as $0.42 \mathrm{~nm}$ (decane/water), $2.13 \mathrm{~nm}$ (decane/water/anion), $1.16 \mathrm{~nm}$ (decane/water/non-ion), $2.13 \mathrm{~nm}$ (decane/water/zwitterion), and $4.29 \mathrm{~nm}$ (decane/water/gemini). Density profile analysis shed light on the approach of the oil-water interface by anionic C5Pe asphaltene molecules [43]. Fig. 2 shows that because of the different densities of oil and water, an obvious oil-water interface with a thickness of 4-6 nm was formed. In addition, starting from $60 \mathrm{ns,} \mathrm{C5Pe} \mathrm{molecules} \mathrm{tended} \mathrm{to} \mathrm{move} \mathrm{toward} \mathrm{the} \mathrm{interface,} \mathrm{and}$ the peak density rapidly increased in the 60-120 ns stage, which indicated that more molecules approached the interface. After $120 \mathrm{~ns}$, the density increase was gentle, and the system reached a stable equilibrium state.

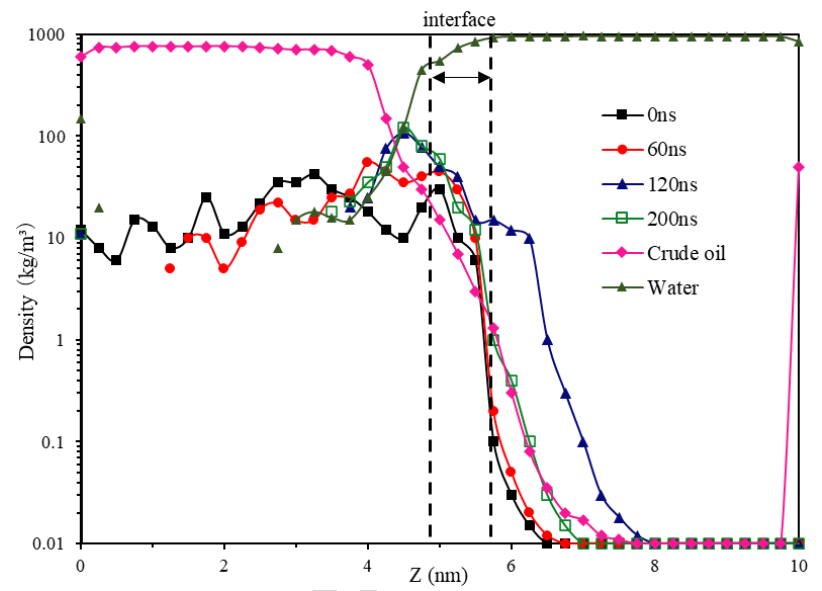

Fig.2 Z-direction density distribution of molecules in the oil-water system (ns is the unit of time scale, $1 \mathrm{~ns}=10^{-9} \mathrm{~s}$ ) [43]

\subsubsection{Interface formation energy}

The interface formation energy (IFE) can be used as a measure of interface stability [44]. For produced oil-water emulsions with surfactants, IFE can be calculated as

$$
I F E=\frac{E_{\text {total }}-\left(n E_{\text {sur }}+E_{\text {oil-water }}\right)}{n}
$$

where $E_{\text {total }}$ is the total energy of the surfactant after the establishment of an equilibrium at the interface, $E_{\text {sur }}$ is the energy of a single surfactant molecule, $E_{\text {oil-water }}$ is the total energy of the oil-water interface system without the surfactant, and $n$ is the number of surfactant molecules.

In the presence of a surfactant, the total energy of the interface decreases, and IFE is negative, with high absolute IFE values denoting increased interface stability and thus favoring interface formation. Kunieda et al. [45] probed light oil/water, pure hydrocarbon/water, and heptane-toluene mixture/water systems by MD simulations, revealing that weak hydrogen bonding between aromatic rings and water protons near the interface reduced the formation energy of the oil-water interface and resulted in an interfacial tension difference, thus favoring the accumulation of aromatic hydrocarbons at the interface. At the same time, hydrogen bond was also the reason why the interfacial tension between aromatic hydrocarbon and water was lower than other hydrocarbons and water.

\subsubsection{Radial distribution function}

As in the case of other liquids, the structure of produced emulsions can be characterized by a radial distribution 
function [45], $g_{i j}(r)$, which represents the average number of atoms $j$ per unit volume located at a distance $r$ from the target atom $i$ and is a basic function for describing liquid and disordered systems. Specifically, this function reflects the probability of finding another particle in the space of radius $r \sim r+d r$ with one particle as the center and describes the distribution of distance between one particle and other surrounding particles. In the radial distribution function diagram, if there is a short-range order in the range of $r$, a peak will appear. The sharper the peak is, the more ordered the arrangement between molecules is, the stronger the connection between molecules is, and the stronger the interaction mode is. Fig. 3 shows the aggregation degree of wax molecules under variable-intensity magnetic fields revealed with the help of the radial distribution function [46]. The peak of the radial distribution function gained intensity with increasing magnetic field intensity $(\mathrm{T})$, which indicates that the aggregation degree of wax molecules concomitantly increased. In other words, because of the effect of magnetic force, the distance between wax molecules decreased, and wax molecules accumulated together to form a dense structure.

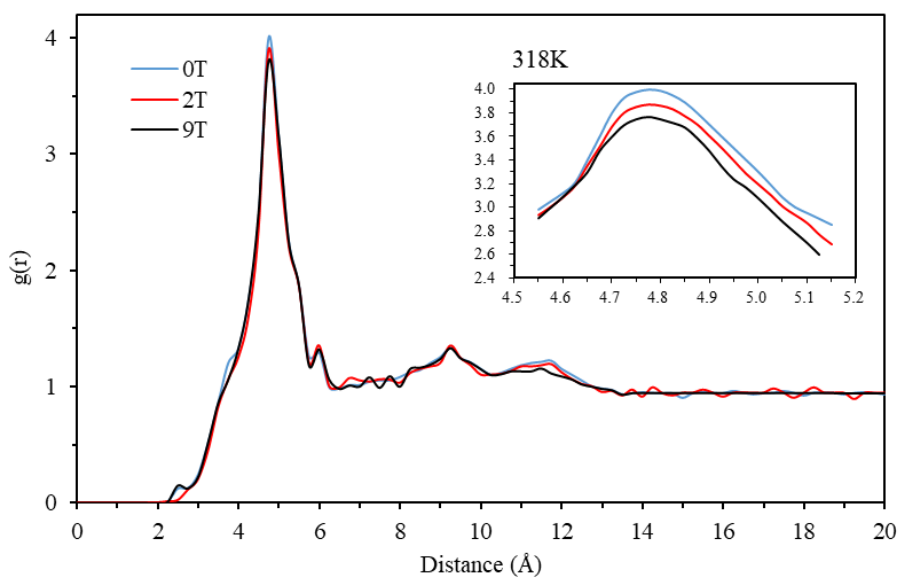

Fig. 3 Radial distribution function of wax molecules under variable-intensity magnetic fields [46]

\subsubsection{Diffusion coefficient}

The aggregation structure and dynamic properties of each molecule can significantly affect the microstructure of an oil-water emulsion system. The aggregation characteristics of each molecule can be characterized by mean square displacement (MSD) and diffusion coefficient $(D)$ [47]:

$$
D=\frac{1}{6 N} \lim _{t \rightarrow \infty} \frac{\mathrm{d}}{\mathrm{d} t} \sum_{i=1}^{N}\left\{\left[r_{i}(t)-r_{i}(0)\right]^{2}\right\}
$$

where, $N$ is the number of diffusing molecules in the system, and the differential term $\lim _{t \rightarrow \infty} \frac{\mathrm{d}}{\mathrm{d} t} \sum_{i=1}^{N}\left\{\left[r_{i}(t)-r_{i}(0)\right]^{2}\right\}$ is the ratio of mean square displacement to time.

The behaviors of sodium dodecyl benzene sulfonate (SDBS), dodecyl trimethyl ammonium bromide (DTAB), nonyl phenol ethoxylate (NPE), and dodecyl dimethyl betaine (Betaine) at the oil-water interface were simulated to compare the vertical MSDs of petroleum molecules in each system and characterize the oil washing performance of these surfactants [47]. The slope of MSD curve indicates the migration ability of petroleum molecules in the vertical direction. The larger the slope is, the stronger the migration ability is, which means the higher the oil washing rate of the surfactant is. Fig. 4 indicates the order of oil washing rate of several different types of surfactants which is SDBS > Betaine $>$ NPE $>$ DTAB. 


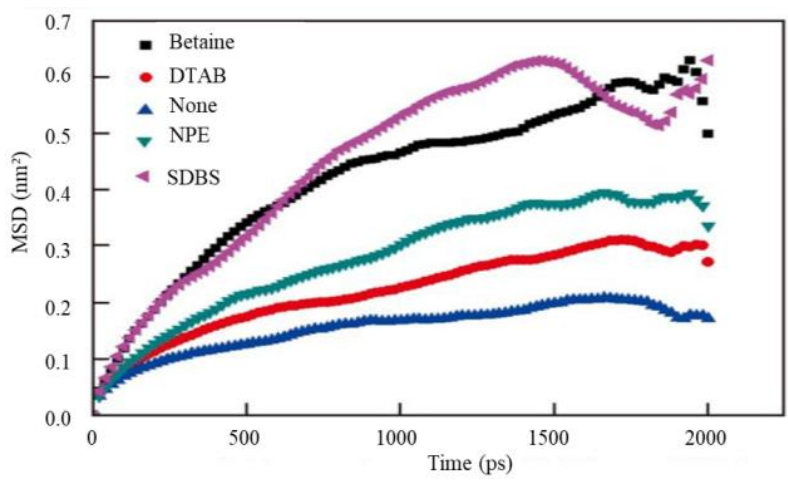

Fig. 4 MSDs of oil molecules along the Z-direction in the presence of different surfactants [47]

Taking the waxy crude oil with 10\% water volume fraction as an example, as shown in Fig. 5, in order to analyze the role of different operating parameters on the diffusion behavior of wax molecules at the oil-water interface, the relationship between diffusion coefficient $(D)$ and system pressure under different temperature conditions was established in our previous study [37]. The diffusion coefficient values indicate that the diffusion coefficient of waxy molecules increase continuously with the increase in relaxation pressure. When it reaches a certain pressure, the variation trend slows down gradually. This can be attributed to the fact that the mobility of waxy molecules increases with the increase in relaxation pressure, then leads to the increase in diffusion ability. When the pressure reaches to a certain value, the binding degree between waxy molecules and other molecules enhances, and the diffusion capacity close to the limit. Under the same pressure and with the increase in the relaxation temperature, the diffusion coefficient increase. In addition, the thermal movement of waxy molecules increases with the increase in temperature, so the diffusion ability will be enhanced accordingly, and the variation trend of diffusion coefficient intensified.

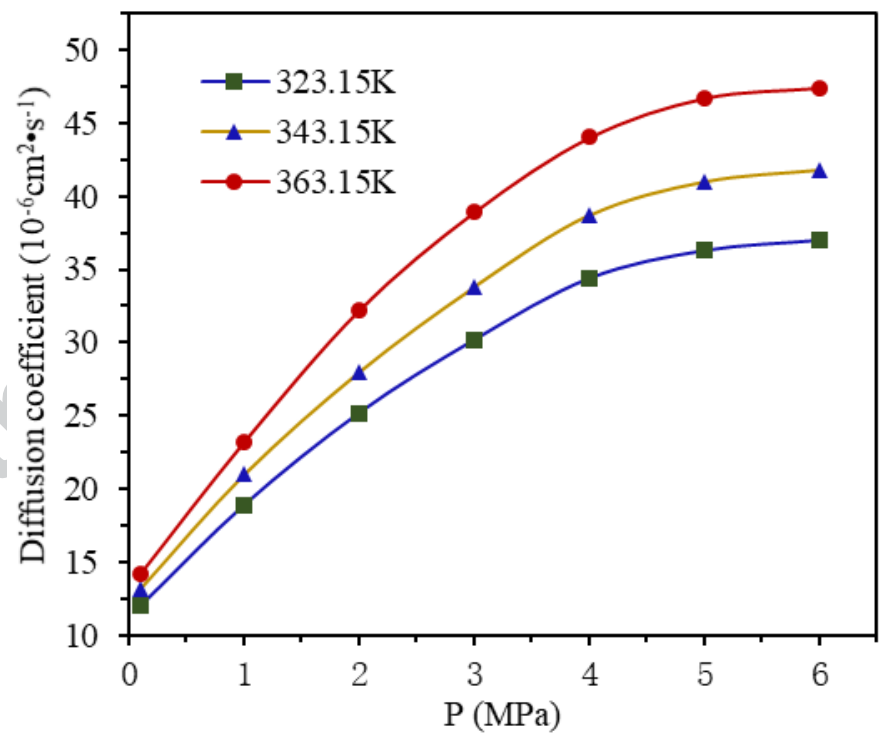

Fig. 5 Diffusion coefficient versus pressure under different temperature [37]

\section{Characterization of oil-water interface behavior}

Since the 1990s, the rapid development of MD has resulted in its increased application in the field of petroleum science. Gamba et al. [48] performed a pioneering investigation and used the "sandwich" structure of the two-layer SDS molecular intercalated water molecular layer to study the hydration force between the surfactant and water molecules, providing a useful reference for the later simulation of interface systems. Afterwards, scholars gradually used this "sandwich" structure to establish emulsion models, including W/O emulsion, O/W emulsion, and even multilevel 
emulsion. For example, the given oil and water molecules are arranged in a lattice, placing water layer in the middle and oil layers on both sides would represent the W/O emulsion, the reverse placement is assigned as the O/W emulsion. Baaden et al. [49,50] reported the microscopic behavior of tributyl phosphate at the oil-water interface and in oil-water mixtures based on MD simulation. In China, because of the wide application of the ASP flooding technology in oilfield development, many scholars have employed MD to probe the aggregation morphology of surfactants at different concentrations [51,52], focusing on the design and application of surfactants for oil displacement and revealing surfactant behavior at the oil-water interface [53-55]. Meanwhile, an MD method was used to simulate the micelle shape, structure size, and other properties of sodium dodecyl benzene sulfonate in oil-water systems at different concentrations [56].

In view of the limitations imposed by numerous conditions in the simulation of complex systems, the most common method is to simplify a given complex system, study the effects of one or two factors, and finally compare the simulation results with experimental data. For example, Li et al. [57] simplified the oil component to chloroform when studying the role of SDBS in oil-water interface separation by MD, revealing that in the absence of SDBS, the oil and water phases can be quickly separated to reach equilibrium and form an obvious oil-water interface. However, in the presence of SDBS, the separation time significantly increases, and SDBS molecules are orderly arranged at the interface to form a 0.8 -nm-thick interfacial film. In addition, the formation of hydrogen bonds between SDBS and water inhibited the migration of water molecules near the interface, while water molecules far from the interface were more prone to migration and diffusion. In their study of the effect of magnetic field on the aggregation behavior of wax molecules in waxy crude oil, Chen et al. [46] used a simplified crude oil model with $\mathrm{C}_{26} \mathrm{H}_{54}$ and $\mathrm{C}_{38} \mathrm{H}_{78}$ as wax molecules.

To date, a general idea of studying oil-water interface behavior by molecular dynamics simulation method has been formed. And the revelation of the mechanism of oil-water interface stability also has been confirmed with the understanding formed by macro scale research. Moreover, MD has been used to further describe the interactions between phases, molecular adsorption, and droplet coalescence/separation. However, the MD-based characterization of the thickness of the oil-water interfacial film, description of the arrangement and aggregation behavior of multi-type molecules at the oil-water interface, and the explanation of the stability of the oil-water interface in the complex produced emulsions formed during ASP flooding pose certain problems. First, the complexity of crude oil components highlights the need for model simplification, although one should avoid overly simple models containing a single type of molecule or those containing molecules that do not match the actual crude oil components. Second, the stability of crude oil emulsions is influenced by internal and external phase components and external environmental factors. Thus, future work should consider oil and water phase components as the main internal factors and temperature and pressure as the main external factors influencing oil-water interface behavior. Finally, the interfacial behavior of surfactants or copolymers in the water phase has so far been the focus of MD research. However, because of the application of various stimulation measures in engineering practice, crude oil emulsions often contain numerous high-molecular-weight polymers, the effective micro-scale characterization of their arrangement and aggregation at the oil-water interface is complicated. Thus, the selection of suitable characterization techniques capable of affording results consistent with those of macroscopic research should be concerned in the future research. The corresponding relationship between macroscopic description and microscopic MD simulation related to the characterization techniques can be summarized in Table 1.

Table 1 Corresponding relation of macroscopic description and microscopic MD simulation.

\begin{tabular}{lll}
\hline \multirow{2}{*}{ Items } & Characterization techniques & \\
\cline { 2 - 3 } & Macroscopic description method & Microscopic MD simulation method \\
\hline Interfacial stability & Interfacial tension test & Interface formation energy \\
& Viscosity test & Radial distribution function \\
Thickness and strength of interfacial film & Optical microscope test & Density distribution
\end{tabular}


Dissolution and diffusion of polar Spectrophotometric test components
Diffusion coefficient

Interface formation energy

Radial distribution function

\subsection{Role of asphaltenes and resins in determining interface stability}

In most cases, carboxylic asphaltenes can be deprotinated into anionic carboxylic asphaltenes. Gao et al. [43] studied the distribution of such asphaltenes at the oil-water interface, showing that because of hydrogen bonding, neutral asphaltenes accumulated in the oil phase to form a face-to-face stacking structure, while deprotonated ones could form face-to-face and T-shaped II stacking structures and be gradually adsorbed from the oil phase to the oil-water interface. According to the energy change, this process can be divided into three stages. First, some anionic asphaltenes form hydrogen bonds to water molecules and are adsorbed on the oil-water interface. Then, more anionic asphaltenes are adsorbed on the oil-water interface via non-covalent interactions. Finally, a face-to-face stacking structure is formed and arranged at the oil-water interface.

Khalaf and Ali [58] simulated the aggregation behavior of three different asphaltenes in a mixture of $n$-heptane and o-xylene using MD and accounting for the effects of van der Waals, hydrogen bonding, and electrostatic interactions on asphaltene aggregation. The action mechanism was found to depend on the number and length of asphaltene molecular chains, the number and type of heteroatoms, and the number of aromatic nuclei. For example, the number of hydrogen bonds in various asphaltene molecules was different, the electrostatic interaction (repulsive vs. attractive) between asphaltene molecules was different.

The aggregation behavior of asphaltenes and resins was studied by MD simulation to explore the influence of their interaction on emulsion stability [59]. The simulation system included asphaltene only, resin only, and asphaltene + resin cases. Analysis of typical radial distribution functions revealed the existence of face-to-face aggregation, accumulation, and many kinds of side-to-side aggregation and accumulation of asphaltene and resin molecules in each system. Among them, face-to-face aggregation and accumulation were the most important form of interaction between asphaltene molecules. Compared with that of the single asphaltene system (Fig. 6(a)), the peak of the single resin system (Fig. 6(b)) was smaller, and the difference between the peak and the other distance (section after the peak) was also smaller. These results revealed that the distribution of aggregation and accumulation structures of resin molecules was random, and the interaction between molecules was weak. In the asphaltene-resin system, the difference between the peak intensity (Fig. 6(c)) and the other distances was the smallest under the same conditions of face-to-face aggregation and accumulation, which indicated that the distribution of the aggregation and accumulation structures of the asphaltene-resin system was more random, and the molecular interaction was weaker than the above two cases. These two types of aggregation and accumulation behavior resulted in the formation of a "fence" structure on the oil droplet surface. This structure wrapped the droplet to form a protective film and stabilize the oil-water interface. In addition, the $\mathrm{N}, \mathrm{O}$, and $\mathrm{S}$ atoms in asphaltene and resin molecules formed weak hydrogen bonds with water molecules to stabilize emulsions.
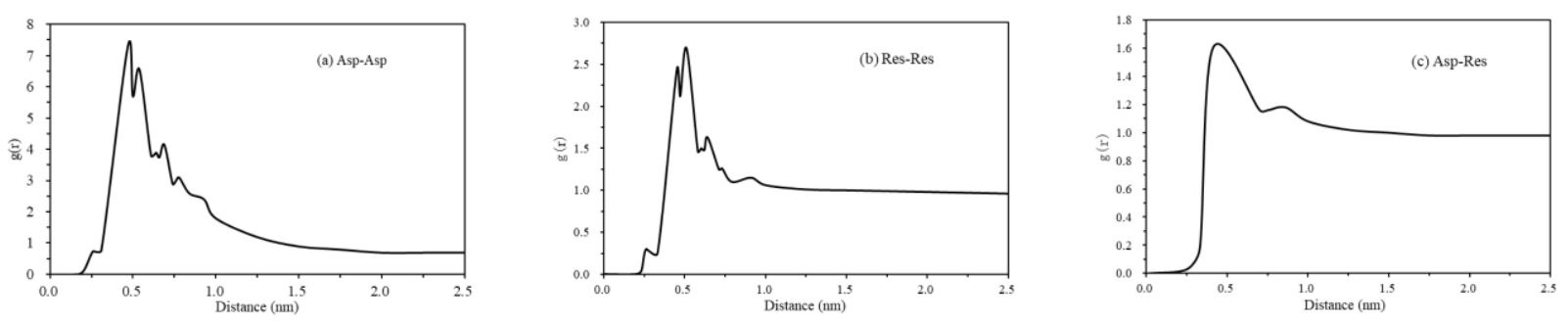

Fig. 6 Radial distribution functions for (a) asphaltene-asphaltene, (b) resin-resin, and (c) asphaltene- resin systems [59]

\subsection{Role of surfactants and polymers in determining interface stability}


MD simulation was used to explore the oil-water interface properties of four surfactants (sodium dodecyl sulfonate, SDS, sodium dodecyl benzene sulfonate, and sodium dodecyl diethyl sulfate) with the same alkyl tail and different head groups [60-62]. The mechanism of molecular structure influence on interfacial tension was revealed by analyzing the interface formation energy, interface thickness, and the water-surfactant interaction, which provided a useful reference for the design of efficient surfactant structures. Li et al. [40] studied the influence of $\mathrm{pH}$ on the interface performance of an oil/water/surfactant system. The heavy oil component was simplified to octadecane, and two surfactants, ADS and ADHA, were introduced. As these surfactants can engage in reversible conversion reactions at different $\mathrm{pH}$, the influence of $\mathrm{pH}$ was simplified to that of ADS:ADHA molar ratio on interface performance. With increasing $\mathrm{pH}$, the tension of the oil-water interface first decreased and then sharply increased, which was ascribed to the decreasing distance of the surfactant head group-water interaction with increasing surfactant concentration. This decreasing distance made the water molecules closely approach the surfactant layer, the polarity of which also brought greater miscibility, so that the surfactant molecules were more widely distributed. At the same time, the interface formation energy continued to increase, and miscibility was weakened.

Niu et al. [63] studied the influence of the EO-PO copolymer demulsifier on emulsion stability by combining interfacial tension tests with Brewster angle microscopy imaging and MD simulation. C5Pe asphaltene and xylene were selected as oil components in this simulation. The results revealed that the EO-PO copolymer featured strong interfacial activity and could replace most C5Pe asphaltene molecules at the interface. At the same time, MD simulation showed that copolymer molecules adsorbed on the oil-water interface through hydrogen bonding to form a surface barrier preventing the further adsorption of C5Pe. Thus, EO-PO molecules destroyed the interfacial film formed by C5Pe and favored the coalescence of emulsion droplets. In another work, MD simulation was used to explore the salt resistance of polymers and surfactants and probe the interactions of polymers with ionic species, revealing functional groups on the polymer chain interacting with ions [64]. Moreover, the MD simulation of a complex system of a salt-resistant polymer and a salt-resistant surfactant was used to explore the type and mode of interaction between these compounds, revealing that cations had more significant effects on the molecular behavior of polymers and surfactants than anions.

\section{Conclusions}

Asphaltenes and resins (crude oil components) as well as surfactants and polymers (present in the water phase) can exhibit different interfacial adsorption behaviors, affecting the strength of the oil-water interfacial film and the stability of crude oil emulsions. Thus, the use of MD to simulate the influence mechanism of these factors and thus enrich the theory of oil-water emulsification at different levels (from macro- to microscale) is a task of high importance.

MD simulation offers the advantage of microstructure characterization and has therefore become an important method of probing interactions between phases. This simulation allows one to obtain thickness changes of oil-water interfacial film, probe the arrangement and aggregation behavior of various molecules at the oil-water interface, and determine the stability of the oil-water interface through the quantitative characterization of the density distribution of emulsion systems, the radial distribution function of emulsion structures, the formation energy of the oil-water interface, and the molecular diffusion coefficient.

Future work in the field of MD simulation of oil-water emulsification/demulsification systems should focus on circumventing the limitations of model simplification and single-factor simulation, integrating the characteristics of emulsion internal and external phases, and accounting for external environmental factors. Furthermore, understanding the potential role of bubbles on produced emulsion stability in chemical flooding is also attractive.

\section{Acknowledgments}

This work presented in this paper was financially supported by the PetroChina Innovation Foundation (Grant No. 2019D-5007-0501) and the State Key Program of National Natural Science Foundation of China (Grant No. 51534004). 


\section{References}

[1] Rui, Z., Peng, F., Ling, K.; Chang, H.; Chen, G.; Zhou, X.: Investigation into the performance of oil and gas projects. J. Nat. Gas Sci. Eng. 38, 12-20 (2017). https://doi.org/10.1016/j.jngse.2016.11.049.

[2] Rui, Z.; Li, C.; Peng, F.; Ling, K.; Chen, G.; Zhou, X.; Chang, H.: Development of Industry performance metrics for offshore oil and gas project. J. Nat. Gas Sci. Eng. 39, 44-53 (2017). https://doi.org/10.1016/j.jngse.2017.01.022.

[3] Wang, Z.; Bai, Y.; Zhang, H.; Liu, Y.: Investigation on gelation nucleation kinetics of waxy crude oil emulsions by their thermal behavior. Journal of Petroleum Science and Engineering, 181, 106230 (2019). https://doi.org/10.1016/j.petrol.2019.106230.

[4] Supee, A.; Idris, A.K.: Effects of surfactant-polymer formulation and salinities variation towards oil recovery. Arabian Journal for Science and Engineering. 39(5), 4251-4260 (2014). https://doi.org/ 10.1007/s13369-014-1025-7.

[5] Anganaei, H.; Pourabdollah, K.; Rostami, A.: Experimental improvement of nano-enhanced oil recovery using nano-emulsions. Arabian Journal for Science and Engineering. 39(8), 6453-6461 (2014). https://doi.org/10.1007/s13369-014-1258-5

[6] Mohsenatabar, F.; Derakhshan, A.; Shadizadeh, S.R.: An investigation into surfactant flooding and alkaline-surfactant-polymer flooding for enhancing oil recovery from carbonate reservoirs: Experimental study and simulation. Energy Sources, Part A: Recovery, Utilization and Environmental Effects. 40(24), 2974-2985 (2018). https://doi.org/10.1080/15567036.2018.1514439

[7] Pal, N.; Saxena, N.; Mandal, A.: Characterization of alkali-surfactant-polymer slugs using synthesized gemini surfactant for potential application in enhanced oil recovery. Journal of Petroleum Science and Engineering. 168, 283-300 (2018). https://doi.org/10.1016/j.petrol.2018.05.026.

[8] Xiao, H.Y.; Zhen, Z.; Sun, H.; Cao, X.; Li, Z.; Song, X.; Cui, X.; Liu, X.: Molecular dynamics simulation of anionic surfactant at the water/n-alkane interface. Acta Physico-Chimica Sinica. 26(2), 422-428 (2010). https://doi.org/10.3866/PKU.WHXB20100216.

[9] Li, K.; Liu, Y.; Yan, H.; Liu, M.: Molecular dynamics simulation of self-assembly behavior of SDBS/BMAB on the oil-water interface. Journal of Liaocheng University (Natural Science Edition). 32(6), 71-75 (2019). https://doi.org/ 10.19728/j.issn1672-6634.2019.06.011.

[10] Bendjaballah, M.; Canselier, J.P.; Oumeddour, R.: Optimization of oil-in-water emulsion stability: Experimental design, multiple light scattering, and acoustic attenuation spectroscopy. Journal of Dispersion Science and Technology. 31(9), 1260-1272. (2010). https://doi.org/10.1080/01932690903224888.

[11] Bansbach, P.L.: Treating emulsions produced by thermal recovery operations. In: SPE California Regional Meeting. (1965). https://doi.org/10.2118/1328-MS

[12] Kilpartrick, P.K.: Water-in-crude oil emulsion stabilization: Review and unanswered questions. Energy \& Fuels. 26(7), 4017-4026 (2012). https://doi.org/10.1021/ef3003262.

[13] Kale, S.N.; Deore, S.L.: Emulsion micro emulsion and nano emulsion: A review. Systematic Reviews in Pharmacy. 8(1), 39-47 (2017). https://doi.org/10.5530/srp.2017.1.8

[14] Wang, Z.; Lin, X.; Rui, Z.; Xu, M.; Zhan, S.: The role of shearing energy and interfacial Gibbs free energy in the emulsification mechanism of waxy crude oil. Energies. 10(5), 721 (2017). https://doi.org/10.3390/en10050721.

[15] Umar, A.A.; Saaid, I.B.M.; Sulaimon, A.A.; Pilus, R.B.M.: A review of petroleum emulsions and recent progress on water-in-crude oil emulsions stabilized by natural surfactants and solids. Journal of Petroleum Science and Engineering. 165, 673-690 (2018). https://doi.org/10.1016/j.petrol.2018.03.014.

[16] Wong, S.F.; Lim, J.S.; Dol, S.S.: Crude oil emulsion: A review on formation, classification and stability of water-in-oil emulsions. Journal of Petroleum Science and Engineering. 135, 498-504 (2015). https://doi.org/10.1016/j.petrol.2015.10.006.

[17] Jha, P.; Mahto, V.; Saxena, V.: Study the effects of xanthan gum and aluminium stearate on the properties of oil-in-water emulsion drilling fluids. Arabian Journal for Science and Engineering. 41(1), 143-153 (2016). https://doi.org/10.1007/s13369-015-1646-5.

[18] Dicharry, C.; Arla, D.; Sinquin, Anne.; Graciaa, A.; Bouriat, P.: Stability of water/crude oil emulsions based on interfacial dilatational rheology. Journal of colloid and interface science. 297(2), 785-791 (2006). https://doi.org/10.1016/j.jcis.2005.10.069.

[19] Fingas, M.; Fieldhouse, B.; Mullin, J.: Water-in-oil emulsions results of formation studies and applicability to oil spill modelling. Spill Sci. Technol. Bull. 5 (1), 81-91 (1999). https://doi.org/10.1016/S1353-2561(98)00016-4.

[20] Chen, S.; Zhang, L.; Xie, L.; Liu, Q.; He, J.; Mantila, C.A.; Vandenberg, F.G.A.; Zeng, H.: Surface interaction of water in oil emusion 
droplets with interfacially active asphaltenes. Langmuir. 33(5), 1265-1274 (2017). https://doi.org/10.1021/acs.langmuir.6b04265.

[21] Andersen, S.I.; Birdi K.S.: Aggregation of asphaltenes as determined by calorimetry. Journal of Colloid and Interface Science. 142(2), 497-502 (1991). https://doi.org/10.1016/0021-9797(91)90079-N.

[22] Wang, Z.; Yu, X.; Li, J.; Wang, J.; Zhang, L.: The use of biobased surfactant obtained by enzymatic syntheses for wax deposition inhibition and drag reduction in crude oil pipelines. Catalysts. 6(5), 61 (2016). https://doi.org/10.3390/catal6050061.

[23] Torrealba, V.A.; Johns, R.T.: Coupled interfacial tension and phase behavior model based on micellar curvatures. Langmuir. 33(47), 13604-13614 (2017). https://doi.org/10.1021/acs.langmuir.7b03372.

[24] Hakiki, F.; Maharsi, D.; Marhaendrajana, T.: Surfactant-polymer coreflood simulation and uncertainty analysis derived from laboratory study. Journal of Engineering and Technological Sciences. 47(6), 706-724 (2015). https://doi.org/10.5614/j.eng.technol.sci.2015.47.6.9.

[25] Xin, X.; Zhang, H.; Xu, G.; Tan, Y.; Zhang, J.; Lv, X.: Influence of CTAB and SDS on the properties of oil-in-water nano-emulsion with paraffin and span 20/Tween 20. Colloids and Surfaces. A: Physicochemical and Engineering Aspects. 418, 60-67 (2013). https://doi.org/10.1016/j.colsurfa.2012.10.065.

[26] Azadeh, M.; Mohammad, R.R; Abdolhossein, J.: Effect of a cationic surfactant as a chemical destabilization of crude oil based emulsions and asphaltene stabilized. Journal of Chemical \& Engineering Data. 57(6), 1689-1699 (2012). https://doi.org/10.1021/je2013268.

[27] Li, M.; Xu, M.; Lin, M.; Wu, Z.: The effect of HPAM on crude oil/water interfacial properties and the stability of crude oil emulsions. Journal of Dispersion Science and Technology 28 (1), 189-192 (2007). https://doi.org/10.1080/01932690600992829.

[28] Yang, Q.; Xin, X.; Wang, L.; Lu, H.; Ren, H.; Tan, Y.; Xu, G.: Modification of the stability of oil-in-water nano-emulsions by polymers with different structures. Colloid and Polymer Science. 292(6), 1297-1306 (2014). https://doi.org/10.1007/s00396-014-3185-0.

[29] Meller, A.; Stavans, J.: Stability of emulsion with nonadsorbing polymers. Langmuir. 12(2), $301-304$ (1996). https://doi.org/10.1021/la950440h.

[30] Qiu, J.: Stability of the oil-water emulsion formed during amphiphilic polymer flooding. Petroleum Science and Technology. 31(2), 142-147 (2013). https://doi.org/10.1080/10916466.2011.603006.

[31] Xu, J.; Liu, H.; Wang, Z.: Effect of polymer concentration on the properties of the crude oil-water emulsion. Petroleum Science and Technology. 36(24), 2057-2063 (2017). https://doi.org/10.1080/10916466.2018.1471497.

[32] Rothlisberger, U.: Magic and mysteries of modern molecular dynamics simulation. In: Domenicano A., Hargittai I. (eds) Strength from Weakness: Structural Consequences of Weak Interactions in Molecules, Supermolecules, and Crystals. NATO Science Series (Series II: Mathematics, Physics and Chemistry) 68, 121-141 (2002). https://doi.org/10.1007/978-94-010-0546-3_7.

[33] Haruna, K.; Saleh, T.A.; Obot, I.B.: Atomistic simulation: A unique and powerful computational tool for corrosion inhibition research. Arabian Journal for Science and Engineering. 44(1), 1-32 (2019). https://doi.org/10.1007/s13369-018-3605-4.

[34] Elaheh, S.; Effat, J.; Seyyed, M.A.H.; Tahereh, S.; Ehsan, Z.: Molecular dynamics simulation of boron nitride nanotube as a drug carrier. Arabian Journal for Science and Engineering. 39(9), 6737-6742 (2014). https://doi.org/10.1007/s13369-014-1228-y.

[35] Alasiri, H.: A molecular dynamics simulation probe of the solubility parameters of supercritical water and methanol. Arabian Journal for Science and Engineering. 44(12), 9911-9917 (2019). https://doi.org/10.1007/s13369-019-03957-w.

[36] Perilla, J.R.; Goh, B.C.; Cassidy, C.K.; Liu, B.; Bernardi, R.C.; Rudack, T.; Yu, H.; Wu, Z.; Schulten, K.: Molecular dynamics simulations of large macromolecular complexes. Current Opinion in Structural Biology. 31, 64-74 (2015). https://doi.org/10.1016/j.sbi.2015.03.007.

[37] Gan, Y.; Cheng, Q.; Wang, Z.; Yang, J.; Sun, W.; Liu, Y.: Molecular dynamics simulation of the microscopic mechanisms of the dissolution, diffusion and aggregation processes for waxy crystals in crude oil mixtures. Journal of Petroleum Since and Engineering. 179, 56-69 (2019). https://doi.org/10.1016/j.petrol.2019.04.059.

[38] Li, B.; Liu, S.; Fang, S.; Chen, Y.; Hu, W.: Progress in molecular docking and molecular dynamics simulation. Journal of Comparative Chemical. 3(1), 1-10 (2019). https://doi.org/10.12677/CC.2019.31001.

[39] Cisneros-Dévora, R.; Cerón-Camacho, R.; Soto-Castruita, E.; Perez-Alvarez, M.; Ramirez-Perez, J.F.; Oviedo-Roa, R.; Servin-Najera, A.G.; Buenrostro-Gonzalez, E.; Martinez-Magadan, J.M.; Zamudio-Rivera, L.S.: A theoretical study of crude oil emulsions stability due to supramolecular assemblies. Colloids \& Surfaces A: Physicochemical and Engineering Aspects. 567, 121-127 (2019). 
https://doi.org/10.1016/j.colsurfa.2019.01.045.

[40] Li, H.; Dai, C.; Wu X.: Developing new recyclable and pH-sensitive amphiphile for heavy oil emulsion and demulsification: A molecular dynamics study. In: 7th International Field Exploration and Development Conference (IFEDC). (2017). https://doi.org/10.1007/978-981-10-7560-5_97.

[41] Du, Z.; Chen, Z.: Molecular dynamics simulation study on behaviors of liquid 1,2-dichloroethane under external electric fields. Journal of Zhejiang University (Science). 4(1), 70-76 (2003). https://doi.org/10.1631/jzus.2003.0069.

[42] Shi, P.; Zhang, H.; Lin, L.; Song, C.; Chen, Q.; Li, Z.: Molecular dynamics simulation of four typical surfactants at oil/water interface. Journal of Dispersion Science and Technology. 39(9), 1258-1265 (2018). https://doi.org/10.1080/01932691.2017.1392319.

[43] Gao, F.; Xu, Z.; Liu, G.; Yuan, S.: Molecular dynamics simulation: The behavior of asphaltene in crude oil and at the oil/water interface. Energy Fuels. 28(12) 7368-7376 (2014). https://doi.org/10.1021/ef5020428.

[44] Liu, C.; Zhang, X.; Tan, N.; Zeng, D.: Thickness and fractional dimension number of the liquid-vapor interface. Journal of Engineering Thermophysics. 25, 562-564 (2004). https://doi.org/10.1023/B:JOGO.0000006653.60256.f6.

[45] Kunieda, M.; Nakaoka, K.; Liang, Y.; Miranda, C.; Ueda, Akira.; Takahashi, Satoru.; Okabe, Hiroshi.; Matsuoka, Toshifumi.: Self-accumulation of aromatics at the oil-water interface through weak hydrogen bonding. Journal of the American Chemical Society. 132(51) 18281-18286 (2011). https://doi.org/10.1021/ja107519d.

[46] Chen, X.; Hou, L.; Li, W.; Li, S.; Chen, Y.: Molecular dynamics of magnetic field influence on waxy crude oil. Journal of Molecular Liquids. 249, 1052-1069 (2018). https://doi.org/10.1016/j.molliq.2017.11.101.

[47] Jiang, R.; Luo, J.; Bai, R.; Jiang, B.; Zhou, G.: Molecular dynamics simulation on behavior of common surfactants at the oil/water interface in complex systems. Chemical Journal of Chinese Universities. 38(10), 1804-1812 (2017). https://doi.org/10.7503/cjcu20170242.

[48] Gamba, Z.; Hautman, J.; Shelley, J.C.; Klein, M.L.: Molecular dynamics investigation of a newton black film. Langmuir. 8(12), 3155-3160 (1992). https://doi.org/10.1021/la00048a050.

[49] Baaden, M.; Burgard, M.; Wipff, G.: TBP at the water-oil interface: The effect of TBP concentration and water acidity investigated by molecular dynamics simulations. Journal of Physical Chemistry B. 105(45), 11131-11141 (2001). https://doi.org/10.1021/jp011890n.

[50] Baaden, M.; Wipff, G.; Bemy, F.: The chloroform / TBP / aqueous nitric acid interfacial system: A molecular dynamics investigation. Journal of Molecular Liquids. 90(1-3), 1-9 (2001). https://doi.org/10.1016/S0167-7322(00)00174-4.

[51] Shi, J.; Lu, K.; Yuan, S.: Molecular dynamics simulation of alkyl benzene sulfonate at the oil-water interface. Journal of Shandong University. 42(2), 77-82 (2012). https://doi.org/10.6040/j.issn.1672-3961.2012.02.013.

[52] Yan, H.; Cui, P.; Liu, C.; Yuan, S.: Molecular dynamics simulation of pyrene solubilized in a sodium dodecyl sulfate micelle. Langmuir. 28(11), 4931-4938 (2012). https://doi.org/10.1021/la300146s.

[53] Tan, J.; Cao, X.; Li, Y.; Song, X.; He, X.; Cui, X.; Li, Z.: Investigate of synergistic effects mechanism of mixed surfactants at the oil/water interface. Chemical Journal of Chinese Universities. 30(5), 949-953 (2009). https://doi.org/10.1115/MNHMT2009-18287.

[54] Hu, X.; Li, Y.; Sun, H.; Song, X.; Li, Q.; Cao, X.; Li, Z.: Effect of divalent cationic ions on the adsorption behavior of zwitterionic surfactant at silica/solution interface. Journal of Physical Chemistry. B. 114(27), 8910-8916 (2010). https://doi.org/10.1021/jp101943m.

[55] Zhao, H.; Sun, H.; Qi, N.; Li, Y.: Understanding of the foam capability of sugar-based nonionic surfactant from molecular level. Colloids \& Surfaces A: Phys. 551, 165-173 (2018). https://doi.org/10.1016/j.colsurfa.2018.05.010.

[56] Gao, J.; Ren, Y.; Ge, W.: Molecular dynamics simulation of effect of salt on the compromise of hydrophilic and hydrophobic interactions in sodium dodecyl sulfate micelle solutions. Chinese Journal of Chemical Engineering. 17(4), 654-660 (2009). https://doi.org/ 10.1016/s1004-9541(08)60259-1.

[57] Li, Z.Q.; Guo, X.L.; Wang, H.Y.; Li, Q.H.; Yuan, S.L.; Xu, G.Y.; Liu, C.B.: Molecular dynamics simulation of anionic surfactant aggregation at the oil/water interface. Acta Physico-Chimica Sinica. 25(1), 6-12 (2009). https://doi.org/10.3866/PKU.WHXB20090102.

[58] Khalaf M.H.; Mansoori, G.A.: A new insight into asphaltenes aggregation (an MD simulation study). Journal of Petroleum Science and Engineering. 162, 244-250 (2018). https://doi.org/10.1016/j.petrol.2017.12.045.

[59] Song, S.; Zhang, H.; Sun, L.; Shi, J.; Cao, X.; Yuan, S.: Molecular dynamics study on aggregating behavior of asphaltene and resin in emulsified heavy oil droplets with sodium dodecyl sulfate. Energy Fuels. 32(12), 12383-12393 (2018). 
https://doi.org/10.1021/acs.energyfuels.8b03258.

[60] Xu, J.; Zhang, Y.; Chen, H.; Wang, P.; Xie, Z.; Yao, Y.; Yan, Y.; Zhang, J.: Effect of surfactant headgroups on the oil/water interface: An interfacial tension measurement and simulation study. Journal of Molecular Structure. 1052, 50-56 (2013). https://doi.org/10.1016/j.molstruc.2013.07.049.

[61] Wang, Z.; Lin, X.; Yu, T.; Zhou, N.; Zhong, H.; Zhu, J.: Formation and rupture mechanisms of visco-elastic interfacial films in polymer-stabilized emulsions. J. Dispers. Sci. Technol. 40, 612-626 (2019). https://doi.org/10.1016/j.molstruc.2013.07.049.

[62] Zhong, H.; Yang, T.; Yin, H.; Lu, J., Zhang, K., Fu, C.: Role of alkali type in chemical loss and ASP-flooding enhanced oil recovery in sandstone formations. SPE Reservoir Evaluation \& Engineering. 23(2), 431-445 (2020). https://doi.org/10.2118/191545-PA.

[63] Niu, Z.; Ma, X.; Manica, R.; Yue, T.: Molecular destabilization mechanism of asphaltene model compound C5Pe interfacial film by EO-PO copolymer: Experiments and MD simulation. Physical Chemistry C. 123(16), 10501-10508 (2019). https://doi.org/10.1021/acs.jpcc.9b02248.

[64] Hu, X.Y.; Li, Y.; Zhang, H.; He, X.J.; Xue, Y.Z.; Wang, P.; Yang, J.L.: Molecular dynamics simulation of foam films and analysis of foam drainage mechanism. Acta Chimica Sinica. 68(2), 131-135 (2010). https://doi.org/10.1631/jzus.B1000073. 